\title{
Complete reconstruction of anterior segment surgery for a patient with traumatic cataract and iris defects: a case report
}

\section{Zhenyu Wang}

Peking University Third Hospital https://orcid.org/0000-0001-6458-3944

Xuemin Li ( $\nabla$ 13911254862@163.com)

Peking University Third Hospital

\section{Case Report}

Keywords: Traumatic cataract; Traumatic iris defect; Surgery

Posted Date: January 15th, 2019

DOI: https://doi.org/10.21203/rs.2.227/v1

License: (c) (1) This work is licensed under a Creative Commons Attribution 4.0 International License.

Read Full License 


\section{Abstract}

Background: Ocular trauma has relationship with variable damage towards anterior segment or posterior segment. Among them, cornea, iris and lens are vulnerable to injury. For patients with traumatic cataract and other anterior segment trauma $₫$ complete reconstruction of anterior segment surgery can be a good option to restore patients' anterior segment that helps them regain their vision.

Case presentation: We presented a case of traumatic cataract with iris defects after explosive trauma. A surgery was done to fully reconstruct the anterior segment of the patient's left eye including separating posterior synechia of iris, removing cataract and implantation of IOL. Postoperatively, the patient didn't complain about photophobia anymore and the uncorrected visual acuity (UCVA) of the patient reached from counting fingers to 20/25 and remained stable on follow-up at 1 week and 1 month.

Conclusion: Our case suggested the importance of completely reconstruct anterior segment for patients who have traumatic cataract with traumatic iris defects and its benefits for society.

Keywords: Traumatic cataract; Traumatic iris defect; Surgery

\section{Background}

Ocular trauma has relationship with variable damage towards anterior segment or posterior segment [1]. Among them, cornea, iris and lens are vulnerable to injury. In such cases, patient may experience a period of time with extremely low visual acuity and photophobia if they have traumatic cataract with iris defects. According to the survey [2], cataract (including traumatic reasons) is the dominant cause of sociomedical problem i.e., blindness worldwide. Blindness can be devastating physically and economically to patients. Many of them died early [3]. It is a terrific challenge for ophthalmologists to deal with blindness due to traumatic cataract on public stance. The only available treatment is surgery. However, doctors can't guarantee complete recovery of patients' vision. The success of surgery, especially the surgery to deal with traumatic cataract, is based in adequate communication and personalized analysis. For patients with traumatic cataract and other anterior segment trauma $\bigotimes$ complete reconstruction of anterior segment surgery can be a good option to restore patients' anterior segment that helps them regain their vision.

Here we presented a case of traumatic cataract with iris defects after explosive trauma. A surgery was done to fully reconstruct the anterior segment of the patient's left eye including separating posterior synechia of iris, removing cataract and implantation of IOL. Postoperatively, the patient didn't complain about photophobia anymore and the uncorrected visual acuity (UCVA) of the patient reached from counting fingers to 20/25 and remained stable on follow-up at 1 week and 1 month. The report shows the necessity of performing surgeries on patients with complicated traumatic cataract who have visual expectation and its benefits for society. Besides, the report shows the importance of completely reconstruct anterior segment for patients who have gone through severe ocular trauma, especially for patients who have iris defects and may have double pupils if the breaches are not closed. 


\section{Case Presentation}

A 69-year-old man presented with the complaints of blurred vision and photophobia in his left eye for more than 20 years following explosive trauma. The patient reported that the explosion occurred around his left eye 20 years ago and it was unclear that if there was foreign body in his left eye. He gave a history of swelling of his eyelid and inability to open his left eye for 2 days after the trauma. On the $4^{\text {th }}$ day he noticed the defect of his vision and did not come to hospital for treatment. His vision hasn't recovered since then.

A series of ophthalmic examinations were conducted. Best corrected visual acuity (BCVA) of the patient's left eye is counting fingers with full light projection. The intraocular pressure of the left eye was 10 $\mathrm{mmHg}$. Physical examination revealed that the cornea was clear and the depth of anterior chamber was normal. There was a breach on the bottom of the iris and there was posterior synechia as well. The pupil was not round and pupillary reaction to light was absent. Lens showed irregular white opacification which blocked our observation of his fundus (Figure 1).

With blurred vision, the patient was unable to work and experienced a tough life with financial burden. The visual expectation was fully discussed and considered after all the risks of the surgery were explained to the patient. A detailed surgical plan was made and put into practice. The goal of this surgery was to fully reconstruct the anterior segment of the patient's left eye including separating posterior synechia of iris, removing opacified lens and implantation of IOL. After precisely measured, the IOL power calculation was done using SRK-II formula and the appropriate IOL power (HOYA, +20.0 D) was chosen. A $3.2 \mathrm{~mm}$ corneal incision was made using a keratome and a side port was made on the $2 \mathrm{o}$ clock of the limbus. Because of the fibrosis, it was hard to remove the anterior capsule in a round shape. The adhersion of iris and anterior capsule was separated slowly and the anterior capsule was removed carefully. After the auto-l/A device was entered, the residue cortex was aspirated clearly. We found that there was adhersion between anterior and posterior capsule. The residual area of the capsule was large enough to implant the IOL with a single-point suspension on the 12 o' clock of the limbus. In order to prevent double pupils, we sutured the inferior breach of the iris with a single 10-0 nylon suture. The whole surgery lasted for about 15 minutes. The breach of the iris was closed and a round-shaped pupil was formed. The IOL was implanted in the correct position (Figure 2).

On postoperative day 1 , the patient felt comfortable and was not afraid of light anymore. Uncorrected visual acuity (UCVA) of this patient was 20/25. Mild edema of wounded conjunctiva was found. The cornea was clear and there was a little KP. The depth of anterior chamber was normal and the pupil was round. A well-centered IOL was in situ. The patient didn't complain during the postoperative period. On follow-up at 1 week and 1 month, the UCVA were still 20/25 and there was no KP. IOL was stable without decentration.

\section{Discussion And Conclusion}


Ocular trauma involving anterior segment, especially lens and iris, can seriously affect vision, which cause traumatic cataract and traumatic iris defects. It is a terrific challenge for ophthalmologists to deal with blindness due to traumatic cataract with surgical management. The success of surgery is based in adequate communication and personalized analysis. In this case, the patient with traumatic cataract and traumatic iris defects was unable to work and experienced a tough life with financial burden. It is a heavy burden and harmful for society as well. Thus in this situation, patients should not be abandoned and it is necessary to perform surgeries to reconstruct the anterior segment of the patient's eye.

The surgery included two major parts. The first part is removing opacified lens and implantation of IOL. For traumatic cataract patients, the choice of intraocular lens is very important. After accurate preoperative measurements, we chose HOYA IOL as the IOL we implanted in the patient's eye. The HOYA IOL had three-piece form with good elasticity, which could be implanted conveniently, and firmly. Besides, the thin haptics of this IOL effectively avoided repeated friction of the patient's damaged iris.

The second part is separating posterior synechia and closing the breaches of the iris. Iris directly determines the amount of light that enters into the eye. Deficient iris can diminish visual acuity because of increased spherical aberration, glare, photophobia, decreased contrast sensitivity, and decrease depth of focus [4]. Small iris defects can be repaired using microtying suture techniques while large defects need to be replaced by implantable iris material. In 2012, Spitzer M, S, et al. [5] describe a new surgical technique for treating traumatic aniridia with aphakia using a 3-piece acrylic intraocular lens through the haptics to a customized silicone iris prosthesis. In this case, suture of the iris defect effectively improved the visual acuity and photophobia of this patient.

We presented a case of traumatic cataract with iris defects after explosive trauma. We performed a surgery to fully reconstruct the anterior segment of the patient's left eye and improved the visual acuity and photophobia of this patient. Our case suggested the importance of completely reconstruct anterior segment for patients who have traumatic cataract with traumatic iris defects and its benefits for society.

\section{Abbreviations}

UCVA: uncorrected visual acuity

BCVA: Best corrected visual acuity

IOL: intraocular lens

\section{Declarations}

Ethics approval and consent to participate

The authors certify that Peking University Third Hospital Medical Ethics Committee approved all procedures used in this study. The authors have obtained all patients' consents to participate. 
Consent for publication

Patients have given their consents for publication. Written consents were obtained from patients in our study. Patients have given their consents to use their clinical information (including data and material).

Competing interest

None.

Funding

None.

Authors' contribution

ZW analyzed and interpreted the patient's data, and was a major contributor in writing the manuscript. XL performed the surgery. All authors read and approved the final manuscript.

Acknowledgements

None.

\section{References}

1. Maltzman, B. A.,Pruzon, H., Mund, M., L.. A survey of ocular trauma. Surv Ophthalmol. 1976;21(3): 285-290.

2. Gupta, V. B., Rajagopala, M., Ravishankar, B.. Etiopathogenesis of cataract: an appraisal. Indian J Ophthalmol. 2014;62(2): 103-110.

3. World Health Organization. Strategic Plan for Vision 2020: The Right to Sight. Elimination of Avoidable Blindness in the South-East Asia Region, SEA-Ophthal-117, World Health Organization Regional Office for South-East Asia New Delhi. 2000.

4. Weissbart, S. B. and B. D. Ayres. Management of aniridia and iris defects: an update on iris prosthesis options. Curr Opin Ophthalmol. 2016;27(3): 244-249. 
5. Spitzer, M. S., Yoeruek, E., Leitritz, M., A., et al. A new technique for treating posttraumatic aniridia with aphakia: first results of haptic fixation of a foldable intraocular lens on a foldable and customtailored iris prosthesis. Arch Ophthalmol. 2012;130(6): 771-775.

\section{Figures}

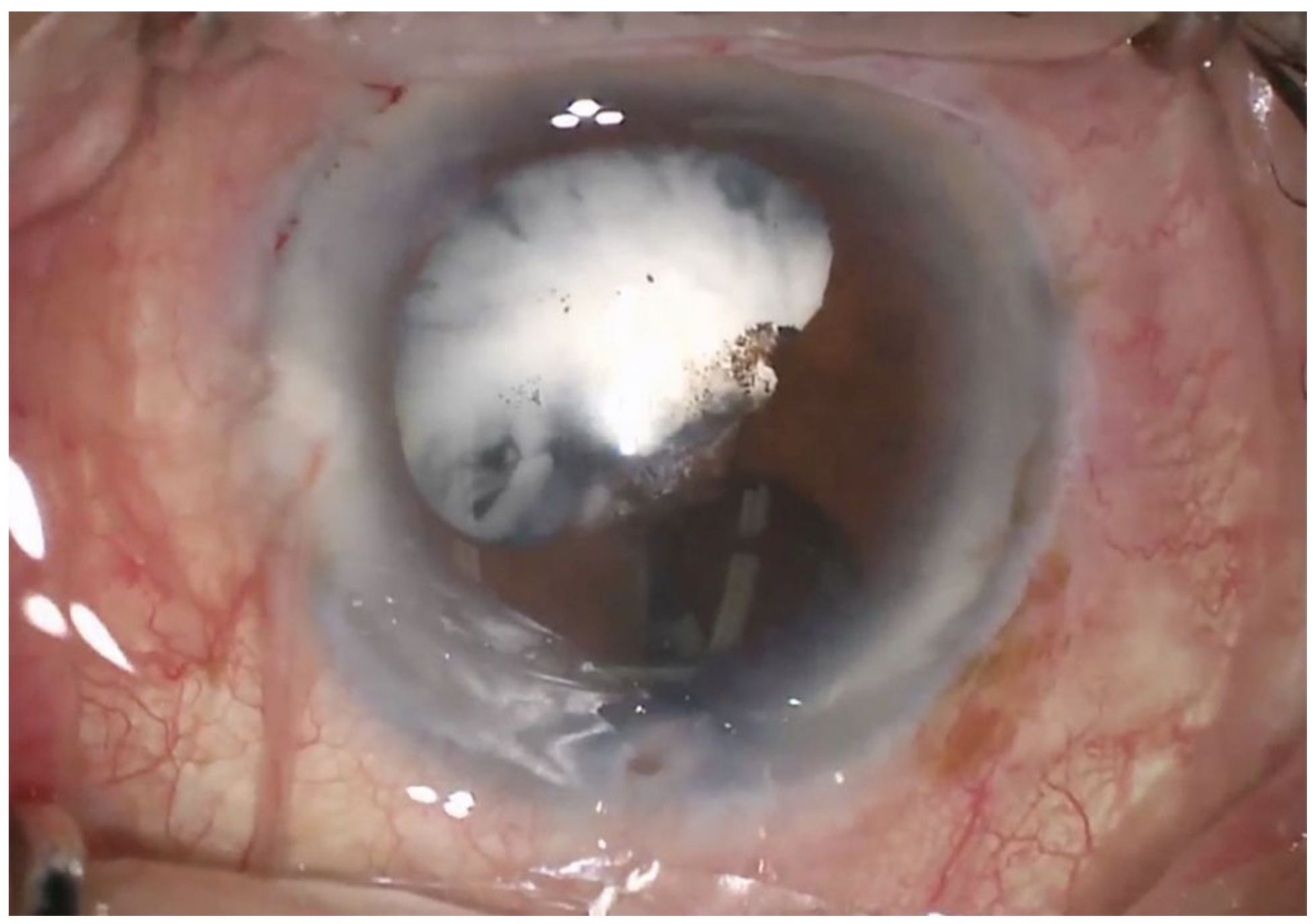

Figure 1

Color photograph of the left eye showing traumatic cataract and iris defect. 


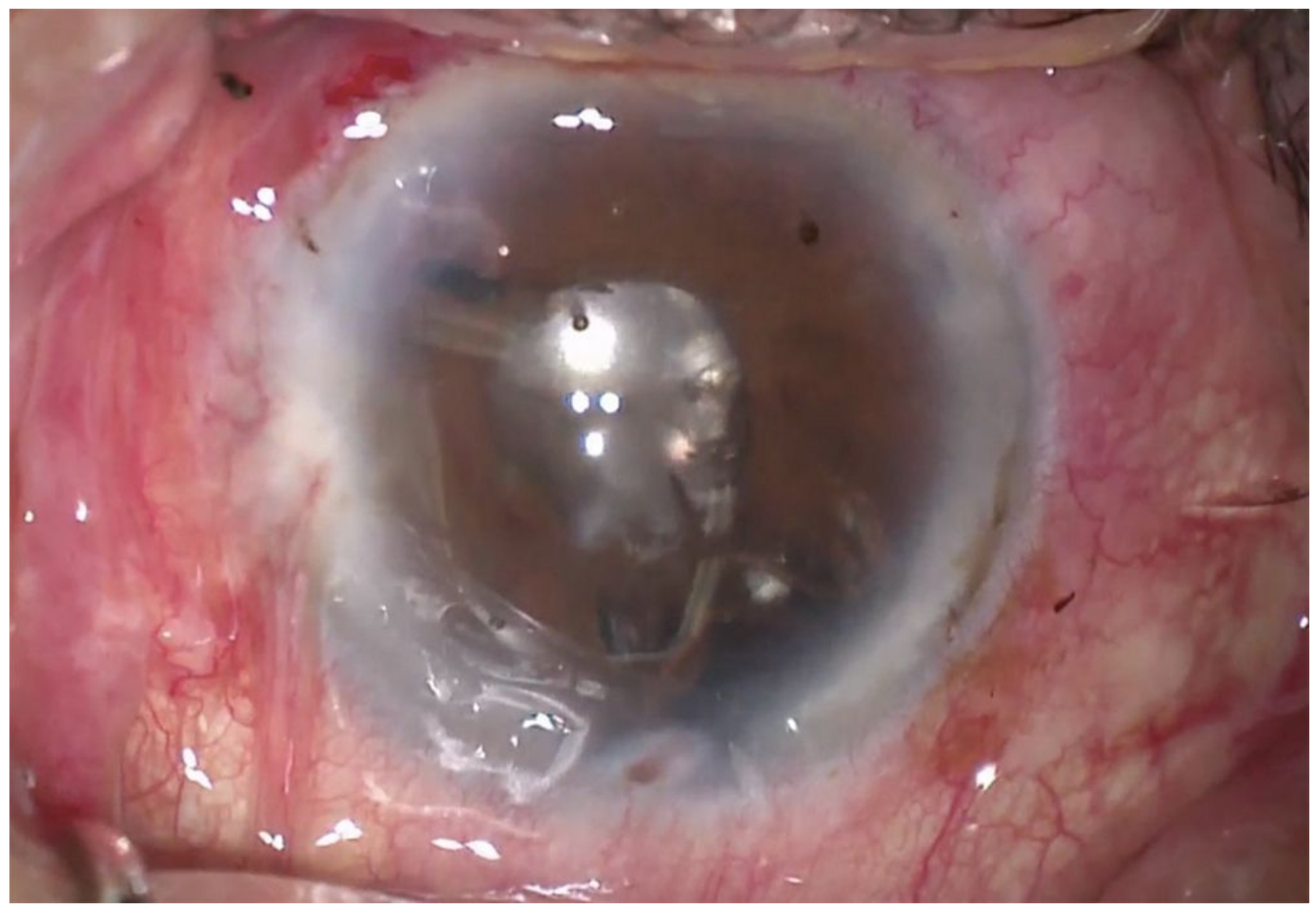

\section{Figure 2}

Color photograph of the left eye postoperatively. The IOL was stable and the breach on the iris was closed.

\section{Supplementary Files}

This is a list of supplementary files associated with this preprint. Click to download.

- supplement1.pdf 